\title{
Artículos
}

\section{Espacio social y clases sociales en Posadas, Argentina}

\author{
Social space and social classes in Posadas, Argentina \\ Elías Gómez*
}

\section{Resumen}

En este artículo se realiza un análisis de las desigualdades sociales urbanas en la ciudad de Posadas, Argentina, a partir de la Encuesta Permanente de Hogares para el tercer trimestre de 2017. Desde un enfoque exploratorio, con conceptos de Pierre Bourdieu y Norbert Elias, se identifican las principales dimensiones del espacio social y las clases sociales vinculadas. La metodología utilizada incluye análisis especifico de correspondencias múltiples, clasificación ascendente jerárquica, agrupamiento de clases y sintesis de etnografias locales. El objetivo principal es una sintesis de las desigualdades sociales urbanas como etapa inicial de la investigación, a la que se incorporarán en etapas subsiguientes nuevas fuentes de datos, técnicas de análisis y etnografias localizadas.

Palabras clave: espacio social; clases sociales; Pierre Bourdieu.

\begin{abstract}
The article proposes an analysis of urban social inequalities in the city of Posadas, Argentina, from the Permanent Household Survey

* Universidad Nacional de Misiones / Consejo Nacional de Investigaciones Científicas y Técnicas. Dirección: Calle Tucumán 1941, CP. 3300, Posadas, Argentina. Correo: 1sgomez001@gmail.com ORCID: https://orcid.org/0000-0002-44689618.
\end{abstract}


for the third quarter of 2017. From an exploratory approach with concepts of Pierre Bourdieu and Norbert Elias, the main dimensions of social space and related social classes are identified. The methodology used includes specific multiple correspondences, hierarchical ascending classification, clustering, and synthesis of local ethnographies. The main objective is a synthesis of urban social inequalities as an initial stage of research; to this synthesis new sources of data, analysis techniques, and localized ethnographies will be incorporated in subsequent stages.

Keywords: social space; social classes; Pierre Bourdieu.

\section{Introducción}

La construcción del espacio social y la identificación de clases sociales es una contribución importante a los estudios urbanos por parte de Bourdieu (1988) e investigadores afines. Retomar esta contribución implica analizar en una primera etapa estructuralista, el volumen global y la composición del capital (Schmitz, Flemmen y Rosenlund, 2018; Hjellbrekke et al., 2007; Borges Pereira, 2018). Siguiendo este enfoque, el objetivo es un análisis exploratorio de las desigualdades sociales en la ciudad de Posadas, Argentina, a partir de la Encuesta Permanente de Hogares (en adelante EPH) del tercer trimestre de 2017. Las preguntas que aborda el artículo son: ¿Cuáles son los ejes de las desigualdades sociales? ¿Qué características generales tienen las clases sociales en el espacio social de la ciudad? Estas preguntas retoman distintos problemas relacionados con la pobreza, las desigualdades de género, las luchas por el poder, las oportunidades económicas, la gentrificación, y la frontera internacional con Paraguay, entre otros. Esta relacionalidad con diferentes temáticas es una ventaja de los análisis multidimensionales, que se ajusta bien a la pretensión de encarar investigaciones menos personales y más colectivas. Los antecedentes más importantes de esta propuesta en Argentina son los análisis sobre el espacio social cordobés (Gutiérrez y Mansilla, 2016), y acerca de los niveles de capitalización de agricultores (Baranger, Niño y Simonetti, 2008; Baranger, 2012). 
Posadas es la capital de la provincia de Misiones, en el noreste de Argentina, y es vecina de la ciudad de Encarnación -capital del estado de Itapúa, en Paraguay-. Posadas fue fundada en 1872 y aún conserva su papel como centro administrativo y comercial de la provincia de Misiones; a su vez, la economía de la provincia se basa principalmente en la industria agropecuaria, agroindustrial y turística. El área de mayor densidad poblacional de Posadas siempre fue el centro de la ciudad; los primeros asentamientos irregulares se emplazaron en la costa del río Paraná y de frente a Encarnación; no obstante, en la actualidad los sectores más empobrecidos se encuentran principalmente en los bordes del área urbana y lejos de la costa del río que rodea al microcentro. La relación entre las ciudades de Posadas y Encarnación siempre fue una parte importante de la vida económica, cultural y política en la región. A propósito de las dinámicas de clases, más adelante se retoman síntesis etnográficas sobre los intercambios transfronterizos entre Posadas y Encarnación, y sobre los desplazamientos urbanos de las clases más desposeídas en Posadas.

\section{Conceptos necesarios}

Bourdieu (1988; 1990) diferencia el momento estructuralista del constructivista en la construcción del espacio social. En el primero analiza la distribución de capitales y elementos de fuerza, y en el segundo, las competencias procesuales que conforman dicha distribución. En este caso, el énfasis recae más en el primer momento, aunque se añaden síntesis de etnografías locales para delinear los rasgos centrales de los procesos constitutivos de las clases.

El espacio social es el mundo social analizado según múltiples dimensiones o múltiples principios de diferenciación. Poseer u ocupar propiedades de estas dimensiones es lo que constituye la fuerza de las personas, lo que las sitúa en determinadas posiciones (Bourdieu, 2000; Baranger, 2012). El concepto de capital refiere a las propiedades más disputadas del espacio social, sobre las cuales existe una intuición compartida respecto a su importancia. A propósito de la $\mathrm{EPH}$, el capital económico incluye, por ejemplo, a los ingresos económicos individuales y los ingresos familiares totales. A su vez, 
el capital cultural contempla los niveles de educación formal, y también la cualificación ocupacional como "saber" menos institucionalizado que la educación formal, pero interiorizado como habilidad o capacidad (Gutiérrez y Mansilla, 2016).

Para Bourdieu (1988), las clases sociales incluyen propiedades relacionadas tanto con la edad, el sexo, la educación formal y el estado civil; por ejemplo, los ingresos económicos, el tipo de ocupación laboral, o la propiedad de los medios de producción. Las clases sociales son realidades multidimensionales menos difusas para el investigador que trabaja "sobre el papel", que para los encuestados que en su vida cotidiana sólo acceden parcialmente a las relaciones que les moldean. Las categorías involucradas con la conformación de las clases sociales de Posadas son también elementos de fuerzas; en otras palabras, son propiedades que pueden ser aprovechadas por sus poseedores para mejorar sus posiciones (Elias 1990; 1999); es el caso, por ejemplo, de la edad de los individuos, el estado civil y las relaciones de parentesco dentro del hogar. El concepto de fuerza señala que las personas se hacen dependientes unas de otras a propósito de propiedades diversas, además de por capitales concretos.

\section{Metodología}

La Encuesta Permanente de Hogares (EPH) tiene cobertura nacional y se realiza cuatro veces al año, por muestreo y en conglomerados urbanos. En este caso se retoman los datos correspondientes al conglomerado urbano de Posadas, Argentina, de manera que las categorías utilizadas corresponden a los lineamientos de esta encuesta (EPH, 2017). La EPH sirve como fuente alternativa de datos frente a investigaciones que de otro modo requerirían grandes equipos de trabajo y amplios presupuestos de ejecución. Los datos de la encuesta se encuentran divididos en dos matrices, las cuales posteriormente fueron combinadas: una correspondiente a los hogares, y la otra a los individuos. Para la EPH (2017), el hogar implica la corresidencia de sus miembros y una serie de gastos compartidos.

Para la selección de los miembros activos en las dinámicas de clases se utiliza el método de bosques aleatorios (Random Forests). 
El bosque generado mediante CART (Classification and Regression Trees) compara y aprende de los árboles de decisiones correspondientes a cada miembro de hogar, y luego procede a listar a los árboles de acuerdo al grado de homogeneidad compartida con miembros de otros hogares. El análisis de bosques aleatorios combina varias medidas para comparar y cortar las ramas de los árboles (el coeficiente de Gini para el valor de homogeneidad, chi-cuadrado para la significación estadística, y la entropía para el grado de desorganización de los datos). ${ }^{1}$ Las variables utilizadas para la comparación de los árboles (de miembros de hogares) corresponden a las relaciones de parentesco, los niveles de educación formal, los ingresos económicos individuales y la calificación ocupacional. Para la clasificación se generan hasta 100 árboles diferentes de hasta 10 niveles, y en el caso de la presencia de datos perdidos se amplía el rango de variables consideradas.

En síntesis, los miembros seleccionados comparten grados de homogeneidad y asociaciones significativas a propósito de sus posiciones de parentesco, niveles de educación formal, ingresos económicos individuales y calificación ocupacional; de esta manera, no se consideran los casos demasiado "raros" o atípicos en el interior de los hogares. A su vez, las variables elegidas para la comparación de los árboles corresponden a elementos señalados como cruciales en las estructuras de las sociedades contemporáneas; es el caso del capital económico, el capital cultural, el capital social y simbólico, y las trayectorias sociales (Bourdieu, 1990; Hjellbrekke et al., 2007).

Con el criterio de selección utilizado se acepta como razonable que algunos hogares provean más miembros activos que otros en la conformación de las clases sociales; que dentro de un mismo hogar puedan cohabitar miembros de diferentes clases; y que la cantidad de miembros activos por hogar en la conformación de las clases no sea la misma en cada una de las clases posteriormente identificadas.

${ }^{1}$ Las aplicaciones y propiedades del algoritmo de bosques aleatorios o random forests son temas de investigación en la actualidad, sobre todo en cuestiones de machine learning y data mining. Para conocer un examen sintético de avances e implicaciones estadísticas, véase Louppe, 2014; Biau, 2012; Medina-Merino y Nique-Chacón, 2017. 
Esta selección tiene sentido si se reconoce que los hogares son apenas clases probables (Bourdieu, 1990) -alentadas por la EPH (2017) desde una perspectiva estatal-y que en su interior existen relaciones de poder difícilmente reducibles a la referencialidad de una sola persona. De manera que la unidad de análisis no son los hogares o los individuos jefes o representantes, sino las relaciones entre miembros de hogares con asociaciones fuertes y compartidas en cuanto a posiciones de parentesco, educación formal, ingresos económicos individuales y calificación ocupacional.

Con los datos de los miembros activos (miembros seleccionados) se realiza un análisis de correspondencias múltiples (ACM) especifico, seguido de una clasificación ascendente jerárquica $(\mathrm{CAJ})$ para el agrupamiento de los miembros en clases. En ciencias sociales estas técnicas de análisis se asocian al trabajo de Bourdieu y al análisis geométrico de datos (Le Roux y Rouanet, 2004; Robson y Sanders, 2009; Bourdieu, 1988). Las principales fortalezas de estos análisis incluyen: el descubrimiento y la exposición visual de relaciones de fuerzas; la exposición de homologías estructurales entre múltiples dimensiones (campos) como la economía, la política y la cultura; la explicación de tomas de posición y luchas entre individuos y clases -sobre todo cuando se incorporan análisis cualitativos en profundidad-; y la identificación de dinámicas sociales, especialmente utilizando técnicas de clasificación (Lebaron, 2009; Hjellbrekke et al., 2007).

El objetivo del ACM es:

mostrar geométricamente las filas y columnas de la base de datos -donde las filas representan a los individuos y las columnas a las categorías de las variables, en un espacio de pocas dimensiones- así las proximidades en el espacio indican similaridades entre categorías e individuos [Le Roux y Rouanet, 2010, p. 8].

Una característica central del ACM es que no presupone la existencia de variables causales o independientes; más bien, expone la estructura de fuerzas entre propiedades e individuos, permitiendo la superposición de categorías suplementarias sobre esta estructura. La descripción del análisis se basa en la lectura de coordenadas, con- 
tribuciones, cosenos y pesos de las categorías, así como de valores propios, además de la representación geométrica de los resultados (Rouanet, Ackermann y Le Roux, 2000; Le Roux y Rouanet, 2004). Para el ACM se consideran las siguientes variables activas: la edad, el sexo, el estado civil, el nivel educativo alcanzado, la calificación y el carácter ocupacional, el monto total de ingresos económicos individuales, el monto total del ingreso familiar, la tenencia de vivienda y terreno, y la cantidad de miembros de hogar (Cuadro 3). A las variables menos definitivas se las considera variables suplementarias, en este caso: tipo de vivienda, ubicación de la vivienda (cerca de basurales o en villa emergencia), lugar de procedencia, cobertura de salud, alfabetismo, intensidad de ocupación, relación de parentesco, tipo de trabajo, y rama de la producción.

\section{El espacio de las propiedades}

Los 565 miembros activos (de 1449 individuos) incluyen principalmente a jefes de hogares, cónyuges e hijos; los primeros mayormente con edades de 36 a 47 años y de 48 a 60 años; los segundos, de 36 a 47 años y 35 años o menos; y los terceros, en general con 35 años o menos (Cuadro 1).

El ACM específico considera 10 variables con 34 categorías activas y 9 variables suplementarias con categorías pasivas; las categorías con bajas frecuencias fueron removidas siguiendo la "regla del 5\%” (Le Roux y Rouanet, 2010, p. 216). El primer eje describe el $55 \%$ de la inercia total según el porcentaje modificado de Benzécri, aunque hay variaciones importantes en el segundo (11.5\%) y tercer eje (8.7\%) (Cuadro 2). De manera que se retoma la lectura de estos tres primeros ejes, responsables del $75.2 \%$ de la inercia total.

1) Sobre los que más tienen (Gráfica 1). El primer eje, del volumen global del capital, distribuye a los más desposeídos hacia la izquierda, y a los más capitalizados en general hacia la derecha del espacio social. Las mayores contribuciones vienen de la calificación y el carácter ocupacional, los ingresos económicos individuales y el nivel educativo; las 15 categorías que sobrepasan las contribuciones medias resumen el $84 \%$ de la varianza del eje. En resumen, en la 
ciudad de Posadas las principales coordenadas de las desigualdades sociales diferencian a los miembros de hogares mejores equipados $\mathrm{y}$ más desposeídos en términos ocupacionales, económicos y educacionales.

\section{Cuadro 1}

Miembros activos según posición de parentesco en el interior del hogar y grupo de edad

\begin{tabular}{|c|c|c|c|c|c|}
\hline \multirow[b]{2}{*}{ Parentesco } & \multicolumn{5}{|c|}{ Edad } \\
\hline & $<=35$ años & 36-47 años & 48-60 años & $61-89$ años & Total \\
\hline Jefe & 71 & 104 & 92 & 33 & 300 \\
\hline Cónyuge & 40 & 64 & 28 & 5 & 137 \\
\hline Hijo & 91 & 15 & 3 & 0 & 109 \\
\hline Yerno & 8 & 2 & 0 & 0 & 10 \\
\hline Nieto & 2 & 0 & 0 & 0 & 2 \\
\hline Padre & 0 & 0 & 0 & 1 & 1 \\
\hline Hermano & 3 & 0 & 0 & 0 & 3 \\
\hline Otro familiar & 2 & 1 & 0 & 0 & 3 \\
\hline Total & 217 & 186 & 123 & 39 & 565 \\
\hline
\end{tabular}

Fuente: Elaboración propia con base en EPH, 2017.

2) Sobre los más cualificados (Gráfica 1). El segundo eje expone mejor la fuerza del carácter y cualificación ocupacional en la composición global del capital. Las coordenadas de las principales propiedades involucran mayormente a la calificación y el carácter ocupacional, la edad, el estado civil, el nivel educativo y el sexo; las trece categorías sobrerrepresentadas de estas variables sintetizan el $74.4 \%$ de la inercia del eje. Las oposiciones de mayor peso involucran a los operativos, con edades de 50 o más años, y separados (cuadrante inferior); y a los empleados en servicios varios y servicios sociales, profesionales y técnicos, y no calificados ocupacionalmente (cuadrante superior). Los segundos, relacionados con categorías suplementarias de actividades domésticas, enseñanza, subocupados, hijo y cónyuge; y los primeros, con los jefes de hogar, rama de producción del comercio, la construcción y la manufactura. 


\section{Cuadro 2}

Nube de varianza y especificaciones sobre los principales ejes del ACM

\begin{tabular}{|c|c|c|c|c|c|}
\hline Ejes & $\begin{array}{c}\text { Varianza eje } \\
\text { (valores } \\
\text { propios) }\end{array}$ & $\begin{array}{c}\% \\
\text { varianza } \\
\text { explicada }\end{array}$ & $\begin{array}{c}\% \\
\text { acumulado } \\
\text { varianza } \\
\text { explicada } \\
\end{array}$ & $\begin{array}{c}\% \\
\text { modificado } \\
\text { Benzécri }\end{array}$ & $\begin{array}{c}\text { Ejes } \\
\text { retenidos } \\
\text { criterio } \\
\text { Kaiser } \\
\end{array}$ \\
\hline 1 & 0.32 & 10.0 & 10.0 & 55.0 & $X$ \\
\hline 2 & 0.19 & 6.0 & 15.9 & 11.5 & $X$ \\
\hline 3 & 0.18 & 5.5 & 21.5 & 8.7 & $X$ \\
\hline 4 & 0.17 & 5.3 & 26.7 & 7.1 & $X$ \\
\hline 5 & 0.16 & 4.9 & 31.7 & 5.4 & $X$ \\
\hline 6 & 0.14 & 4.4 & 36.1 & 3.3 & $X$ \\
\hline 7 & 0.14 & 4.3 & 40.4 & 2.9 & $X$ \\
\hline 8 & 0.13 & 4.1 & 44.5 & 2.2 & $X$ \\
\hline 9 & 0.12 & 3.7 & 48.2 & 1.1 & $X$ \\
\hline 10 & 0.11 & 3.5 & 51.7 & 0.8 & $X$ \\
\hline 11 & 0.11 & 3.4 & 55.1 & 0.6 & $X$ \\
\hline 12 & 0.11 & 3.3 & 58.4 & 0.5 & $X$ \\
\hline 13 & 0.11 & 3.3 & 61.7 & 0.4 & $X$ \\
\hline 14 & 0.10 & 3.1 & 64.8 & 0.2 & $X$ \\
\hline 15 & 0.10 & 3.0 & 67.8 & 0.1 & $X$ \\
\hline 16 & 0.10 & 3.0 & 70.7 & 0.1 & $X$ \\
\hline 17 & 0.09 & 2.8 & 73.5 & 0.0 & $X$ \\
\hline 18 & 0.08 & 2.6 & 76.1 & 0.0 & \\
\hline 19 & 0.08 & 2.5 & 78.6 & 0.0 & \\
\hline
\end{tabular}

Nota: Traza de la matriz: 3.214 .

Fuente: Elaboración propia con base en EPH, 2017.

3) Sobre los jóvenes y mayores (sin ilustración). El tercer eje expone mejor la fuerza de las trayectorias sociales en la composición global del capital; la diferencia más notable es aquella entre los más jóvenes y los adultos mayores. Las variables con más contribuciones son la cantidad de miembros de hogar, el nivel educativo, el estado civil y la edad; en conjunto, las trece categorías sobrerrepresentadas describen el $77.8 \%$ de la inercia del eje. De un lado del espacio se ubican aquellos (a) viviendo en hogares con un miembro, separados, 
con ingresos familiares totales de menos de $\$ 14$ mil, empleados en servicios sociales, y profesionales y técnicos, y con 50 años o más. En la otra mitad, (b) los menores de 30 años, viviendo en hogares con cinco y seis miembros, con secundaria completa, y empleados en gestión administrativa.

\section{Gráfica 1}

Análisis de correspondencias múltiples; variables activas y suplementarias

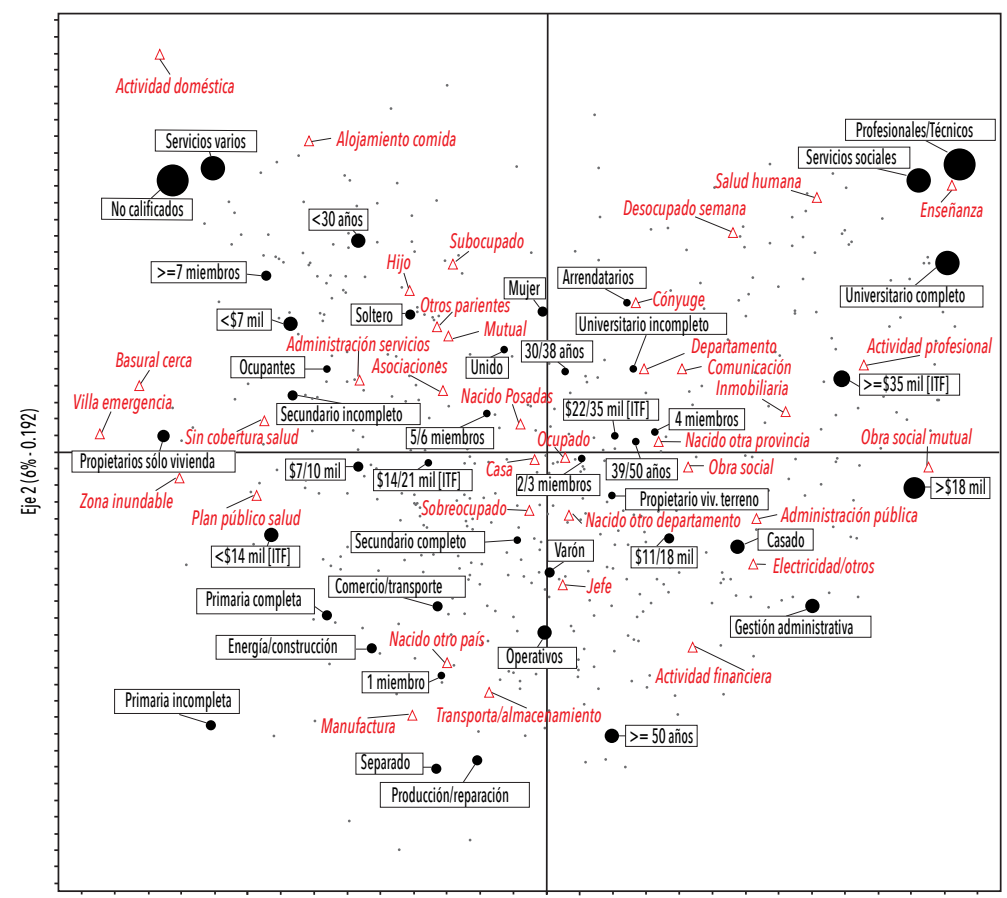

Eje 1 $(10 \%-0.32)$

Fuente: Elaboración propia con base en la EPH, 2017.

En síntesis, las desigualdades sociales en la ciudad de Posadas implican un conjunto de correspondencias entre el volumen global 
de capital, la cualificación ocupacional y las trayectorias sociales. No obstante, en la descripción de las principales diferencias ninguna categoría se ajusta completamente a un solo eje, ya sea en su contribución o peso, sino que aporta en mayor medida a uno y en menor medida a otros. La educación formal, por ejemplo, aporta tanto al primero, como al segundo y tercer ejes, por su estrecha asociación con los ingresos económicos, el carácter ocupacional y las trayectorias sociales (Cuadro 3).

\section{Cuadro 3}

Variables activas, peso y contribuciones a los principales ejes del ACM

\begin{tabular}{|c|c|c|c|c|c|c|}
\hline Variables & $\begin{array}{l}\% \text { peso } \\
\text { relativo }\end{array}$ & Eje 1 & Eje 2 & Eje 3 & Eje 4 & Eje 5 \\
\hline Nivel educativo & 9.9 & 16.9 & 9 & 16.4 & 0.7 & 19.6 \\
\hline Sexo & 10 & 0 & 7.9 & 3.6 & 9.3 & 13.2 \\
\hline Estado civil & 9.7 & 5 & 9.8 & 13.6 & 17.2 & 7.4 \\
\hline $\begin{array}{l}\text { Carácter } \\
\text { ocupacional }\end{array}$ & 9.6 & 18.6 & 23.7 & 11.2 & 13.6 & 19.4 \\
\hline $\begin{array}{l}\text { Calificación } \\
\text { ocupacional }\end{array}$ & 10 & 17.2 & 23.8 & 8.5 & 3.4 & 1.8 \\
\hline $\begin{array}{l}\text { Ingresos totales } \\
\text { familiares }\end{array}$ & 9.6 & 12.3 & 1.4 & 11.5 & 8.8 & 6.3 \\
\hline $\begin{array}{l}\text { Ingresos totales } \\
\text { individuales }\end{array}$ & 9.9 & 17.3 & 3.1 & 1.5 & 11.2 & 4.1 \\
\hline $\begin{array}{l}\text { Cantidad } \\
\text { miembros hogar }\end{array}$ & 10 & 2.9 & 3.5 & 19.9 & 13.1 & 9.7 \\
\hline Edad & 10 & 3.4 & 15.4 & 12.2 & 9.2 & 9.4 \\
\hline $\begin{array}{l}\text { Tenencia } \\
\text { vivienda } \\
\text { y terreno }\end{array}$ & 9.8 & 6.5 & 2.5 & 1.6 & 13.5 & 9.2 \\
\hline
\end{tabular}

Fuente: Elaboración propia con base en la EPH, 2017.

Por otra parte, en el espacio social las distancias entre categorías son distancias sociales; y las cercanías son asociaciones entre propiedades afines. Estas asociaciones, como en un mapa cultural (Bennett et al., 2009), exhiben varios "sectores" si los hacemos coincidir con los extremos del espacio. Así, sobre los ejes 1 y 2 (Gráficas 1 y 2), 
en la mitad derecha del espacio pueden diferenciarse a propiedades y miembros cercanos a los más capitalizados; y en la mitad superior a los más cualificados o profesionalizados. A su vez, en el eje tres la principal diferenciación se da entre las generaciones de los más jóvenes y de los mayores. La distribución de las propiedades en los tres ejes expone los esfuerzos de los más capitalizados, los más cualificados, y los adultos y jóvenes por ocupar "sectores" que les aseguren los recursos más importantes del espacio social. Así, la relacionalidad de cada región del espacio social es inseparable de la constelación de intereses de los miembros activos en la conformación de las clases sociales en Posadas. De manera que el análisis de las propiedades más disputadas del espacio social es la antesala a un análisis de los miembros activos con sus afinidades y distanciamientos de clase.

\section{El espacio de los individuos}

Como se había adelantado al principio, en términos de relaciones de parentesco y cantidad de miembros activos dentro del hogar, las estructuras de las clases son disímiles (Cuadro 4). Basta detenerse en la figura del jefe de hogar. Si bien éstos son los más numerosos entre los miembros activos, la participación de cónyuges, hijos, yernos y demás, son más importantes en unas clases que en otras.

Un examen detallado que incluya etnografías -en otro trabajopodría indagar el papel del sexo y el género, de la feminización y el machismo dentro de los hogares, y sus diferencias según clases sociales. Por ejemplo, de los 300 jefes de hogares considerados en este análisis, 86 son mujeres, pero la categoría de jefe es una clase totalmente etic en sentido antropológico (Harris, 1996). La categoría de jefe o jefa es para la gestión estatal de programas sociales, lo cual no quita que impacte fuertemente en las relaciones de poder dentro de los hogares. En Argentina, la emergencia del "programa jefes y jefas de hogar", activo desde el año 2002, se relaciona estrechamente con un progresivo proceso de feminización del liderazgo en los hogares de las clases populares (Bradshaw, Chant y Linneker, 2018). 


\section{Cuadro 4}

Miembros activos según parentesco y clase social

\begin{tabular}{lrrrrrr}
\hline \multirow{2}{*}{$\begin{array}{c}\text { Parentesco/ } \\
\text { edad }\end{array}$} & $\mathbf{1 / 5}$ & $\mathbf{2 / 5}$ & $\mathbf{3 / 5}$ & $\mathbf{4 / 5}$ & $\mathbf{5 / 5}$ & Total \\
\cline { 2 - 7 } Jefe & 112 & 20 & 49 & 55 & 64 & 300 \\
Cónyuge & 47 & 7 & 34 & 46 & 3 & 137 \\
Hijo & 33 & 10 & 9 & 55 & 2 & 109 \\
Yerno & 3 & 0 & 1 & 6 & 0 & 10 \\
Nieto & 2 & 0 & 0 & 0 & 0 & 2 \\
Padre & 1 & 0 & 0 & 0 & 0 & 1 \\
Hermano & 0 & 0 & 0 & 3 & 0 & 3 \\
Otro familiar & 0 & 0 & 0 & 3 & 0 & 3 \\
Total & 198 & 37 & 93 & 168 & 69 & 565 \\
\hline
\end{tabular}

Fuente: Elaboración propia con base en la EPH, 2017.

No obstante, este artículo enfoca, sobre todo, las asimetrías generales entre los principales miembros activos y el patrimonio de sus hogares; estas asimetrías advierten una gran diversidad si se bosquejan los alcances de las clases sociales en el espacio social. Para ilustrar esta diversidad se realiza entonces una clasificación ascendente jerárquica siguiendo el método de Ward y considerando los primeros 17 ejes (99\% de la inercia acumulada del espacio según el porcentaje modificado de Benzécri) sugeridos por el criterio de Kaiser (Cuadro 2). Conviene recalcar que las clases bosquejadas son clases probables, tal como lo son los hogares identificados por la EPH (2017). Nada evita que en la práctica intervengan significativamente otros factores, por ejemplo, la afectividad devenida de la convivencia, la fuerza de los sentimientos religiosos compartidos o del parentesco y la consanguineidad; este recaudo es necesario debido a que la fuente de datos utilizada es de carácter estatal, y que el análisis propuesto es mayormente estructural, aunque se resumen etnografías locales para bosquejar las interdependencias entre clases.

Mediante la clasificación ascendente jerárquica se identifican entonces cinco grandes clases, aunque para el análisis se retoman solamente los perfiles de las clases de: a) los no calificados en servicios varios (clase 4); b) los operativos de la gestión administrativa (clase 
1); y c) los profesionales de servicios sociales (clase 3); éstas son las clases más distanciadas entre sí (Gráfica 2). El nombre y la descripción de la composición de las clases se basa especialmente en el valor test de las categorías - un valor síntesis de la representación de las categorías en cada clase, de la clase en cada categoría, y de las categorías en la población global-; y también en las elipses de las subnubes de clases (Le Roux y Rouanet, 2010, p. 71).

\section{Gráfica 2}

Clasificación ascendente jerárquica a partir del ACM: clases de no calificados en servicios varios, operativos de la gestión administrativa y profesionales de servicios sociales

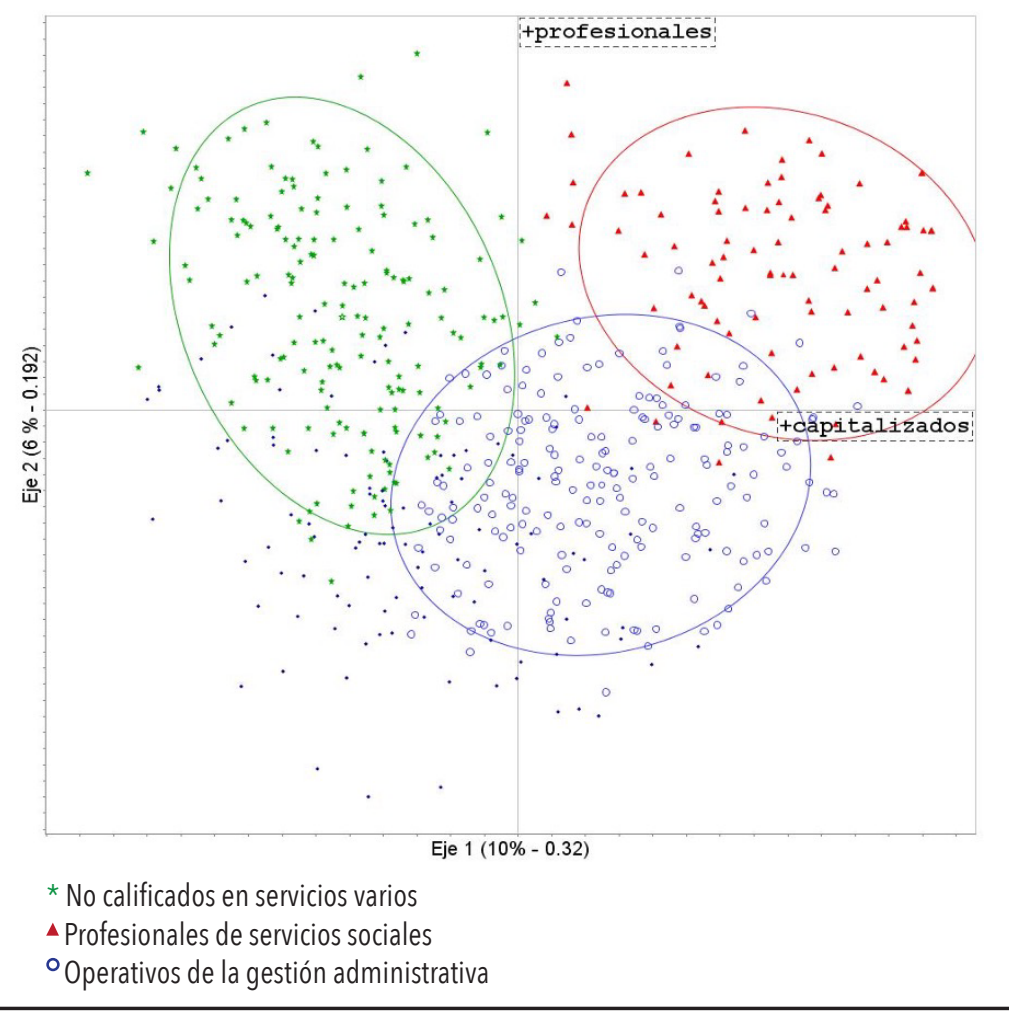

Fuente: Elaboración propia con base en la EPH, 2017. 
a) Clase 4 (Gráfica 2). La clase de los no calificados en servicios varios abarca a 168 personas (29.73\% del total), 97 mujeres y 71 varones; entre ellos sobresalen: los empleados en servicios varios, no calificados, con ingresos individuales iguales o menores de $\$ 7 \mathrm{mil},{ }^{2}$ y con ingresos familiares totales iguales o menores de $\$ 14$ mil, con 35 años o menos, con secundaria incompleta o completa, propietarios sólo de la vivienda, ocupantes viviendo en hogares con cinco o seis miembros [...]. Las principales categorías suplementarias agregan: actividad doméstica, sin cobertura de salud, hijo/a, trabajando en la rama de alojamientos y comidas, en villa de emergencia, subocupados [...].

b) Clase 1. La clase de los operativos de la gestión administrativa incluye a 198 miembros (35.04\% del total), 130 varones y 68 mujeres. Las propiedades sobrerrepresentadas son: operativo, empleado de gestión administrativa, secundaria completa, ingresos individuales iguales de $\$ 11$ a $\$ 18$ mil, o mayores de $\$ 18$ mil, ingresos familiares totales de $\$ 22$ a $\$ 35$ mil, casados, varones, propietario de vivienda y terreno [...]. Las variables suplementarias agregan: con obra social, y empleado en administración pública [...]. Esta clase corresponde en gran medida al sector de los más capitalizados, aunque la subnube de la clase se superpone en pequeñas partes con varias clases.

c) Clase 3. La clase de los profesionales de servicios sociales (profesores, médicos, abogados, arquitectos... [EPH, 2017]) incluye a 93 miembros (16.46\% del total): 37 varones y 56 mujeres. Las categorías sobrerrepresentadas indican: con estudios universitarios completos, empleados en servicios sociales, profesionales o técnicos, con ingresos individuales iguales o mayores a $\$ 18$ mil, ingresos familiares totales iguales o mayores a $\$ 35$ mil, mujeres, casados [...]. Las categorías suplementarias agregan: empleado en la rama de la enseñanza y la salud humana, con obra social, actividad profesional, viviendo en departamentos [...]. Si bien la forma de la subnube de

${ }^{2}$ Como referencia monetaria, el valor de cambio de pesos argentinos a dólares durante el mes de octubre de 2017 oscilaba alrededor de 17.50 pesos por dólar. Es decir, 7000 pesos argentinos serían unos 400 dólares; 14 mil pesos argentinos unos 800 dólares, y así. 
esta clase es un poco dispersa, hacia ella apuntan las clases y fracciones que se esfuerzan por capitalizarse -por ejemplo, la clase de operativos de gestión administrativa-; existe, de hecho, una pequeña superposición de ambas subnubes sobre los ejes 1 y 2 .

\section{Sobre los procesos sociales}

A pesar del resumen esquemático, el momento objetivista es sólo una parte del análisis. Las clases sociales son conformadas en la práctica por competencias que se extienden a lo largo del tiempo. De modo que es necesario enfatizar un par de puntos: a) las clases sociales son constituidas por procesos que abarcan tanto a grupos cercanos como a grupos alejados en el espacio social y geográfico; b) el desarrollo de intereses comunes -materiales o ideales- son una parte importante de la constitución de las clases. En lo que sigue se retoman estos puntos a propósito de etnografías locales y de las clases anteriormente sintetizadas.

\section{a) Interdependencias globales de las clases}

Las síntesis estadísticas de las clases son una abstracción temporal de los resultados de enfrentamientos, alianzas, desacuerdos y acuerdos históricos entre grupos (Bourdieu, 1988; Schmitz, Flemmen y Rosenlund, 2018); el espacio social sintetiza lo que los miembros activos de los hogares pudieron acumular hasta el momento, en términos de ingresos económicos, niveles educativos, ocupaciones, propiedad de vivienda y terrenos, edad, estado civil, y así. No obstante, las condiciones materiales, sentidos culturales y límites de las clases son parte de juegos multipolares -en sentido eliasiano (Elias, 1999)-; es decir, de entramados de dependencias competitivas que reúnen a múltiples grupos. En estos juegos los miembros de hogares pueden, incluso, ser forzados a abandonar el hábitat propio o a migrar hacia otras partes de la ciudad. En Posadas, por ejemplo, en las últimas décadas las clases más desposeídas (como las de los no cualificados y empleados en servicios varios [clase 4]) son alejadas cada vez más del centro de 
la ciudad y del costado del río Paraná. Este caso ilustrativo se vincula con (1) los desplazamientos forzados y (2) los desplazamientos por la gentrificación promovida por agencias gubernamentales, empresas y organizaciones inmobiliarias y comerciales (Brites, 2002, 2014; Barreto, 2004).

1) El gobierno de la provincia de Misiones y la Entidad Binacional Yacyretá (EBY) -principal responsable de la represa hidroeléctrica Yacyretá, construida décadas atrás cerca de la ciudad de Posadas y sobre el río Paraná- impulsaron una serie de relocalizaciones que comenzaron en 1983 e involucraron a más de 25000 personas afectadas por los cambios en el nivel del río. Antes de las relocalizaciones fueron necesarias justificaciones aceptables para la sociedad misionera; éstas enfatizaban la necesidad de energía, de desarrollo regional y nacional, y de infraestructuras necesarias para el crecimiento económico de la región (Brites, 2014; Bartolomé, 1985; Sintes, 2011).

2) Junto a dichas relocalizaciones se realizaron múltiples obras complementarias, entre ellas, la costanera de la ciudad de Posadas, la cual bordea parte del casco céntrico al costado del río Paraná. Esta obra, orientada a la recreación y dispersión, disparó el desplazamiento continuo de las poblaciones desposeídas hacia la periferia de la ciudad. En la actualidad estos desplazamientos son impulsados por la presión de emprendimientos inmobiliarios, de empresas comerciales y por programas gubernamentales orientados al embellecimiento de la ciudad y a la puesta en valor de lugares históricos (Báez y Jaume, 2011; Brites, 2014).

Ambos tipos de desplazamientos, aun con sus diferencias, exponen cómo los procesos constitutivos de las clases más empobrecidas son indisociables de los proyectos de las clases más capitalizadas; y cómo estas últimas no intervienen crudamente sobre las condiciones materiales de las clases más débiles. Antes que nada, se transforman los significados compartidos; es a partir de las transformaciones de sentido que se (re)crean las clases sociales (Thompson, 1984; Bourdieu, 1988). En la actualidad, las clases más desposeídas son desplazadas de la costanera, convencidas de que la misma ya no es una zona para trabajadores marginales, vinculados al río y a la actividad de "paseros" (comercio informal e ilegal) entre Encarnación y Posa- 
das; la costanera es reconocida cada vez más, como una zona para la recreación y la dispersión, para el alquiler de departamentos con vistas al río Paraná, para el disfrute de restaurantes y comidas locales, incluso para la conmemoración monumental de héroes provinciales (Brites, 2014; Jaume, 2018).

\section{b) Desarrollo de intereses comunes y constitución de clases}

Por otra parte, las clases que se esfuerzan por ocupar el sector de los capitalizados y profesionales/ cualificados en el espacio social, difícilmente son "clases dominantes". La evidencia etnográfica encuentra, más bien, a clases enredadas en competencias que afectan de manera imprevista al rumbo de la ciudad. Las contiendas pueden sintetizarse como formando parte de un "campo del poder", que reúne a las clases más capitalizadas. Un caso ilustrativo emerge de las cambiantes dinámicas de las relaciones económicas, políticas y culturales entre la ciudad de Encarnación (Paraguay) y Posadas (Argentina). Ambas ciudades tienen un pasado histórico compartido, aunque las interdependencias de las últimas décadas varían sobre todo debido a las continuas fluctuaciones de la economía argentina. En la actualidad, a propósito de los intercambios económicos transfronterizos, existen oposiciones explícitas entre diferentes sectores de la ciudad (Cossi, 2013; Schiavoni, 1993; Abínzano, 2000).

Así, la Cámara de Comercio y la gestión administrativa asociada al comercio de la ciudad de Posadas (clase probable de operativos de gestión administrativa -clase 1-) suelen exigir mayores controles y multas a los argentinos que compran en Encarnación. Pero también es usual que difícilmente encuentren el apoyo de las clases desposeídas (clases de no cualificados, por ejemplo), o de otras clases capitalizadas, como la clase de profesionales de servicios sociales (profesores, del ámbito de la enseñanza, con estudios universitarios completos [...]-clase 3-). Las ventajas comerciales de la ciudad vecina obligan a los operativos y administrativos ligados al comercio en Posadas a exigir mayores controles en la administración de la frontera internacional. Cuando las ventajas y desventajas se invierten, sucede una tensión similar en Encarnación: la Cámara de Comercio de esa ciu- 
dad presiona al gobierno municipal para que condicione el cruce de compradores paraguayos hacia Posadas, despertando las quejas de otros sectores.

Los intercambios transfronterizos implican, o una oscilación ventajosa en términos de oportunidades económicas, o una oscilación desventajosa que permite la huida de consumidores y contribuyentes; y pueden convivir las dos justificaciones al mismo tiempo, aunque ligadas a la perspectiva de grupos con condiciones sociales diferentes. Estas diferenciaciones pendulares ayudan a refinar los intereses de los grupos en desacuerdo que, eventualmente, pueden transformarse en clases reales. La pregunta implícita sería: ¿Es ventajoso que más argentinos puedan comprar más en Encarnación? La idea que pervive (quizás erróneamente) en las contiendas de este tipo es que, si es ventajoso para unos, no necesariamente lo será para otros. Con el desarrollo de disputas de este tipo, los límites de las clases sociales van conformándose en la práctica porque estos límites no están definidos de antemano por los análisis geométricos estructurales, o por las pretensiones políticas de funcionarios del gobierno. En otras palabras, el carácter interdependiente de los procesos sociales hace que el éxito de las estrategias de determinadas clases dependa en buena medida del fracaso de las estrategias de otras clases (Bourdieu, 1988; Gutiérrez y Mansilla, 2016); acerca de estas interdependencias, de las oposiciones u acuerdos posibles, el aporte de las etnografías localizadas es crucial.

\section{Consideraciones finales}

La EPH (2017) es una fuente de datos abierta y generada por agencias estatales nacionales, con lo cual las categorías que se utilizan tienen sesgos determinados. Por ejemplo, existe una serie de datos ausentes o insuficientes, entre ellos: la propiedad de los medios de producción, los ingresos económicos por inversiones empresariales o financieras, o la desestimación de las multiplicidades de géneros. Estas ausencias son un dato en sí mismo; las categorías estatales se relacionan estrechamente con el desarrollo de la sociedad y las políticas de gobierno (Torrado, 1998; Desròsieres, 2004; 2008). No 
obstante, los sesgos señalados no invalidan la reutilización de los enormes esfuerzos colectivos que supone una encuesta como la EPH para la etapa inicial de una investigación sobre las desigualdades sociales urbanas (Gutiérrez y Mansilla, 2016).

Las preguntas iniciales del articulo fueron: a) ¿cuáles son los principales ejes de las desigualdades sociales en la ciudad de Posadas?, y b) ¿qué características generales tienen las clases sociales en el espacio social de la ciudad?

a) Los principales ejes de las desigualdades sociales en Posadas involucran al volumen global de capital, a la inserción ocupacional y a las trayectorias sociales. En el primer eje destacan los aportes del capital económico (ingresos económico individual y familiar total), el carácter y cualificación ocupacional, y el capital cultural (nivel educativo); las oposiciones fundamentales se dan entre los capitalizados (los que más tienen en general) y los desposeídos (los que menos tienen en general). En el segundo eje, los aportes más fuertes provienen del carácter y cualificación ocupacional, la edad, el estado civil, el nivel educativo y el sexo; las posiciones más diferenciadas corresponden a los operativos, varones, de la rama de comercio y transporte, producción y reparación [...] y a los profesionales o técnicos, empleados en servicios sociales, servicios varios, mujeres [...]. En el tercer eje destacan las generaciones temporales, la cantidad de miembros del hogar, el nivel educativo, el estado civil y la edad. De una parte, aquellos con 30 años o menos, en hogares con cinco o seis miembros, trabajando en gestión administrativa y con secundaria completa; y de otra parte, las generaciones mayores: con 50 años o más, en hogares con sólo un miembro, separados, con ingresos familiares totales de $\$ 14$ mil o menos, y empleado en servicios sociales $[\ldots]$.

b) Los elementos de fuerza que retienen las clases provienen de la relacionalidad de determinadas propiedades en su interior y entre cada una de ellas, pero las características sintéticas abordadas en la descripción de las clases sólo resumen el estado del arte en 2017. Las etnografías sintetizadas a propósito enfatizan las dinámicas interdependientes en las que emergen las clases. No puede entenderse la desposesión de unas clases sin los intereses de los más capitalizados, y viceversa; las etnografías retomadas sobre las poblaciones 
desplazadas hacia el margen del área urbana de Posadas ilustran bien estas dinámicas (Brites, 2014; Bartolomé, 1985; Sintes, 2011; Báez y Jaume, 2011; Jaume, 2018). Por otra parte, la posesión de atributos importantes, como capital económico, cultural o una inserción ocupacional ventajosa, no aseguran la dirección de las demás clases; más bien permiten el acceso a un campo del poder. Poseer u ocupar las propiedades más disputadas del espacio social implica participar de las competencias por la administración de recursos cruciales para la ciudad. Estas competencias se evidencian, por ejemplo, en etnografías locales sobre la definición de políticas económicas transfronterizas entre la ciudad de Posadas (Argentina) y Encarnación (Paraguay) (Cossi, 2013; Schiavoni, 1993).

\section{Bibliografía}

Abínzano, R. (2000). Mercosur, un modelo de integración. Posadas, Argentina: Universidad Nacional de Misiones.

Báez, A. y Jaume, F. (comps.). (2011). Desarrollo y ciudadanía en Misiones, Argentina: escenarios locales, procesos y política. Posadas, Argentina: Creativa.

Baranger, D. (2012). Epistemología y metodología en la obra de Pierre Bourdieu. Posadas, Argentina: Universidad Nacional de Misiones.

Baranger D., Niño, F. y Simonetti, E. (2008). Construcción de una tipología de los ocupantes de tierras privadas en Misiones. En L. J. Bartolomé y G. Schiavoni (comps.), Desarrollo y estudios rurales en Misiones (pp. 97-122). Buenos Aires: Ciccus.

Barreto, M. (2004). Transformaciones de la vida urbana de Posadas y Resistencia a fines de los años noventa. Un estudio sobre la dimensión simbólico-ideológica del espacio urbano público. (Tesis doctoral en Antropología Social. Posadas, Argentina: Universidad Nacional de Misiones).

Bartolomé, L. (1985). Relocalizados: antropología social de las poblaciones desplazadas. Buenos Aires, Argentina: IDES.

Bennett, T., Savage, M., Silva, E., Warde, A., Gayo-Cal, M. y Wright, D. (2009). Culture, class, distinction. Oxon, Inglaterra: Routledge. 
Biau, G. (2012). Analysis of a random forests model. Journal of Machine Learning Research 13, 1063-1095. http://citeseerx.ist. psu.edu/viewdoc/download?doi=10.1.1.413.5188\&rep=rep1\& type $=$ pdf

Borges Pereira, V. (2018). Urban distinctions: Class, culture and sociability in the city of Porto. International Journal of Urban and Regional Research, 42(1), 126-137. https://onlinelibrary.wiley. com/doi/full/10.1111/1468-2427.12532

Bourdieu, P. (1988). La distinción. Criterios y bases sociales del gusto. Madrid, España: Taurus.

Bourdieu, P. (1990). Espacio social y génesis de las clases. En P. Bourdieu, Sociología y cultura (pp. 281-310). Ciudad de México: Grijalbo.

Bourdieu, P. (2000). Espacio social y poder simbólico. En P. Bourdieu, Cosas dichas. Barcelona, España: Gedisa.

Bradshaw, S., Chant, S. y Linneker, B. (2018). Challenges and changes in gendered poverty: The feminization, de-feminization, and re-feminization of poverty in Latin America. Journal Feminist Economics, 25(1), 119-144. https://www.tandfonline.com/doi/full /10.1080/13545701.2018.1529417

Brites, W. (2002). Relocalizaciones: más allá del desarraigo. Estrategias reproductivas en un contexto de vulnerabilidad. Santa Fe, Argentina: El Cid.

Brites, W. (2014). La mega-hidroeléctrica Yacyretá en el vórtice de las reconfiguraciones urbanas. El caso de las ciudades de Posadas, Argentina, y Encarnación, Paraguay. URBS, Revista de Estudios Urbanos y Ciencias Sociales, 4(2), 91-107. https:// ri.conicet.gov.ar/handle/11336/10368

Cossi, C. (2013). Continuidades y discontinuidades en los márgenes. Paso fronterizo Posadas-Encarnación. La Rivada, 1(1), 1-23. http://argos.fhycs.unam.edu.ar/bitstream/handle/123456789 /180/Cossi\%2Bfinal.pdf?sequence=1\&isAllowed=y

Desròsieres, A. (2004). La política de los grandes números. Barcelona: Melusina.

Desròsieres, A. (2008). Analyse des données et sciences humaines: comment cartographier le monde social? Journal Electronique 
d'Histoire des Probabilités et de la Statistique 4(2), 11-18. http:// www.jehps.net/Decembre2008/Desrosieres.pdf

Elias, N. (1990). La sociedad de los individuos. Barcelona, España: Península.

Elias, N. (1999). Sociología fundamental. Barcelona, España: Gedisa. EPH. (2017). Encuesta Permanente de Hogares, tercer trimestre de 2017. Instituto Nacional de Estadística y Censos de Argentina. https://www.indec.gob.ar/

Flemmen, M., Jarness, V. y Rosenlund, L. (2018). Social space and cultural class divisions: The forms of capital and contemporary lifestyle differentiation. The British Journal of Sociology, 69(1), 124-153. https://www.ncbi.nlm.nih.gov/pubmed/28816362

Gutiérrez, A. y Mansilla, H. (comps). (2016). El espacio social de las clases y los instrumentos de reproducción social. Dinámicas de mercado de trabajo, el mercado de las políticas sociales, el mercado escolar y el mercado habitacional. Gran Córdoba. 20032011. Córdoba: Universidad Nacional de Córdoba.

Harris, M. (1996). El desarrollo de la teoría antropológica. Historia de las teorías de la cultura. Madrid, España: Siglo XXI.

Hjellbrekke, J., Le Roux, B., Korsnes, O., Lebaron, F., Rosenlund, L. y Rouanet, H. (2007). The Norwegian field of power anno 2000. Journal European Societies, 9(2), 245-273. https://www. tandfonline.com/doi/full/10.1080/14616690601002749

Jaume, F. (2018). Luchas por la hegemonía: historia y etnografia en la provincia de Misiones, Argentina. Posadas, Argentina: Universidad Nacional de Misiones.

Le Roux, B. y Rouanet, H. (2004). Geometric data analysis. From correspondence analysis to structured data analysis. Dordrecht, Holanda: Kluwer Academic.

Le Roux, B. y Rouanet, H. (2010). Multiple correspondence analysis. Washington D.C.: Sage.

Lebaron, F. (2009). How Bourdieu 'quantified' Bourdieu: The geometric modelling of data. En K. Robson y C. Sanders (eds.), Quantifying theory: Pierre Bourdieu (pp. 11-30). Toronto: Springer.

Louppe, G. (2014). Understanding random forests: From theory to practice. Lieja: University of Liège. https://arxiv.org/pdf /1407.7502.pdf 
Medina-Merino, R. y Ñique-Chacón, C. (2017). Bosques aleatorios como extensión de los árboles de clasificación con los programas R y Python. Interfases, 10, 165-189. https://revistas.ulima.edu. pe/index.php/Interfases/article/view/1775/1828

Robson, K. y Sanders, C. (eds.). (2009). Quantifying theory: Pierre Bourdieu. Dordrecht, Holanda: Springer.

Rouanet, H., Ackermann, W. y Le Roux, B. (2000). The geometric analysis of questionnaires: The lesson of Bourdieu's La Distinction. Bulletin de Méthodologie Sociologique, 65(1), 5-15. https:// www.researchgate.net/publication/240967282_The_Geometric _Analysis_of_Questionnaires_the_Lesson_of_Bourdieu's_La_ Distinction

Savage, M., Devine, F., Cunningham, N., Taylor, M., Li, Y., Hjellbrekke, J., Le Roux, B., Friedman, S. y Miles, A. (2013). A new model of social class? Findings from the BBC's Great British class survey experiment. Sociology, 47(2), 219-250. https://journals. sagepub.com/doi/pdf/10.1177/0038038513481128

Schiavoni, L. (1993). Frágiles pasos, pesadas cargas. Las comerciantes fronterizas de Posadas-Encarnación. Posadas, Argentina: Universidad Nacional de Misiones.

Schmitz, A., Flemmen, M. y Rosenlund, L. (2018). Social class, symbolic domination, and Angst: The example of the Norwegian social space. The Sociological Review, 66(3), 623-644. https: //journals.sagepub.com/doi/pdf/10.1177/0038026117738924

Sintes, L. (2011). Procesos emergentes en el acontecer de una población relocalizada, Posadas, Misiones. En A. Báez y F. Jaume (comps.), Desarrollo y ciudadanía en Misiones, Argentina: escenarios locales, procesos y politica. Posadas, Argentina: Creativa. Thompson, E. (1984). Tradición, revuelta y conciencia de clase. Estudios sobre la crisis de la sociedad preindustrial. Barcelona, España: Grijalbo.

Torrado, S. (1998). Familia y diferenciación social: cuestiones de método. Buenos Aires: Eudeba. 


\section{Acerca del autor}

Elías Gómez es licenciado, maestro y doctor en Antropología Social por la Universidad Nacional de Misiones, y ha realizado estudios de postdoctorado en la Universidad Nacional Autónoma de México. Sus líneas de investigación se centran en los conflictos y las desigualdades sociales, el medio ambiente y la conservación de la naturaleza. En la actualidad es investigador postdoctoral del Consejo Nacional de Investigaciones Científicas y Técnicas de Argentina (Conicet) y profesor-investigador del Centro de Estudios Históricos de la Universidad Nacional de Misiones. ORCID https://orcid. org/0000-0002-4468-9618

Entre sus publicaciones se encuentran:

Gómez, E. (2019). Establecidos y marginados en áreas naturales protegidas: dos casos de estudio en México y Argentina. Letras Verdes. Revista Latinoamericana de Estudios Socioambientales, 26, 5168. https://revistas.flacsoandes.edu.ec/letrasverdes/article/view/ 3825

Gómez, E. (2018). Sobre las configuraciones sociales y los modelos de juego. En F. Jaume (comp.). Luchas por la hegemonía: historia y etnografia en la provincia de Misiones, Argentina. Posadas, Argentina: Editorial Universitaria

Recepción: 30 de julio de 2019. Aceptación: 16 de octubre de 2019. 
